Case report

\title{
Alveolar cyst of the adult: Counterpart of gingival cyst of the adult in edentulous ridge
}

\author{
Maykon Kennedy Schulz ${ }^{a}$, Lucas Ribeiro Teixeira ${ }^{\mathrm{b}}$, Darcy Fernandes ${ }^{\mathrm{a}, *}$, Andreia Bufalino ${ }^{\mathrm{a}}$, \\ Jorge Esquiche Leon ${ }^{\mathrm{b}}$ \\ ${ }^{a}$ Department of Diagnosis and Surgery, São Paulo State University (Unesp), School of Dentistry, Araraquara, SP, Brazil \\ ${ }^{\mathrm{b}}$ Oral Pathology, Department of Stomatology, Public Oral Health, and Forensic Dentistry, School of Dentistry of Ribeirão Preto (FORP/USP), University of São Paulo, \\ Ribeirão Preto, SP, Brazil
}

\section{A R T I C L E I N F O}

\section{Keywords:}

Odontogenic cyst

Gingival cyst of the adult

Lateral periodontal cysts

Edentulous maxilla

Histopathology

\begin{abstract}
A B S T R A C T
A 49-year-old man presenting a nodular swelling on the maxillary alveolar mucosa with 2 months of evolution. An imaginological examination revealed superficial bone resorption. By microscopy, typical features of Gingival cyst of the adult (GCA) were observed. However, in the lesional area there are no teeth and consequently periodontium. Thus, the clinicopathological correlation favored a diagnosis of alveolar cyst of the adult, counterpart of the GCA but on the edentulous maxilla or mandible.
\end{abstract}

\section{Introduction}

Gingival cyst of the adult (GCA) is an uncommon gingival cystic lesion of odontogenic origin, usually found within soft tissues in the anterior region of the mandible and requires some diagnostic considerations. Clinically, GCA presents as a nodular lesion that is commonly found in gingival location, and it is characterized by slow growing and painless [1]. Notably, about $20 \%$ of the GCA cases may exhibit superficial bone resorption [2,3]. In these cases, the main lesion to establish a differential diagnosis is the lateral periodontal cyst (LPC). In fact, GCA is considered the soft tissue counterpart of LPC, which is supported by their histopathological similarities. In this study, we reported an unusual case of GCA on the edentulous maxilla.

\section{Case report}

A 49-year-old man was referred with a nodular lesion on the maxillary alveolar mucosa, with 2 months' duration (Fig. 1). The patient was edentulous for 11 years and reported urothelial carcinoma, which was diagnosed 5 years ago. The imaginological evaluation demonstrated a superficial bone resorption on the alveolar ridge in close association with the lesional area (Fig. 2). The needle aspiration verified a cystic nature of the lesion, revealing a non-bloody serous fluid. An excisional biopsy was proposed and during the surgical procedure the lesion was easily separable from adjacent normal-appearing bone, being completely removed. The histopathological examination exhibited a large cystic space lined by epithelium of approximately 1-5 cuboidal to flat cell layer thicknesses, being supported by a fibrous capsule. The lesion was unicystic and the connective tissue was free of inflammation. In some areas, the junction between the epithelium and the underlying connective tissue was easily detached. Intraluminal papillary projections were occasional, whereas cellular atypia, mucous and/or ciliated cell metaplasia were not observed. Remarkably, foci of plaques or thickening of the epithelial lining, as well as odontogenic epithelium within the capsule, were detected. Moreover, minor salivary gland lobules were visualized in subepithelial location, all them distant from the cystic lesion. The histochemical study included periodic acid-Schiff, with and without diastase digestion, and mucicarmine stains, which were negative (Fig. 3). The clinicopathological correlation favored a diagnosis of alveolar cyst of the adult (ACA), being considered the counterpart of the GCA but on the edentulous maxilla or mandible. After 4 months of follow-up, the patient does not report any symptom or alteration after the surgical procedure.

\section{Discussion}

Gingival cyst of the adult is an uncommon odontogenic cyst, usually found in the canine and premolar regions of the mandible. This condition frequently appears as a small bluish nodule in the buccal attached gingiva that in some cases may exhibit superficial bone

\footnotetext{
* Corresponding author at: Department of Diagnosis and Surgery, São Paulo State University (Unesp), School of Dentistry, Araraquara. Rua Humaitá, 1680, CEP 14801-903, Araraquara, São Paulo, Brazil.

E-mail address: darcyfernandes@foar.unesp.br (D. Fernandes).
} 


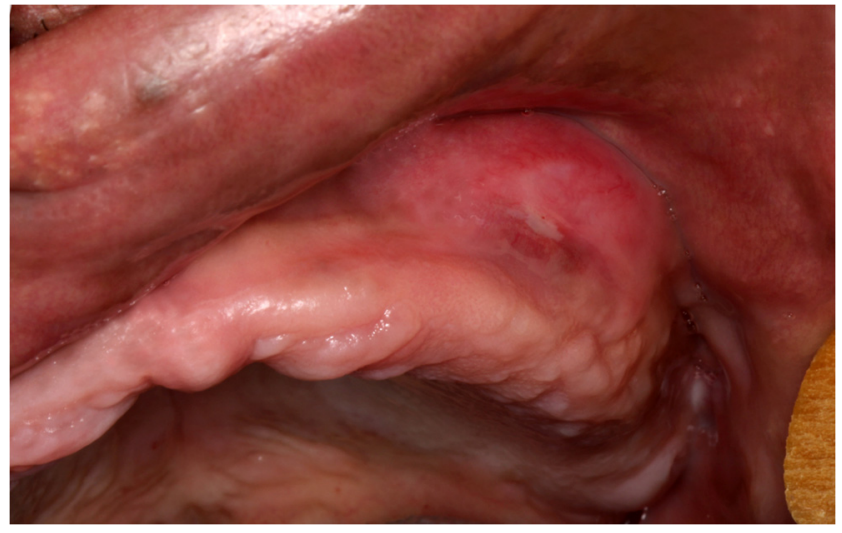

Fig. 1. Clinical appearance of a fluctuant nodular submucosal mass on the left side of the maxillary alveolar mucosa close to premolar region and covered by normal-appearing mucosa.

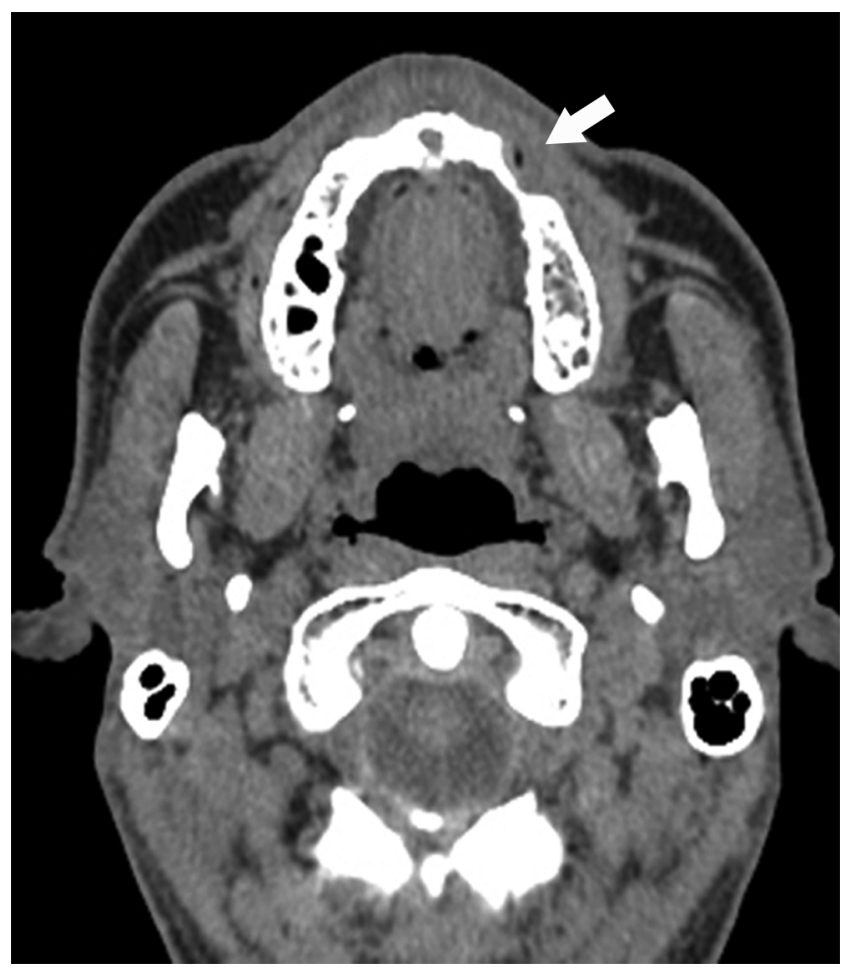

Fig. 2. Axial computed tomography scans reveal a well-defined, superficial bone resorption in the left anterior maxilla (white arrow).

resorption. This lesion usually affects patients between the fifth and sixth decades of life, with a slight female preference. The racial predilection is greater in Caucasians than in Asiatics [2,3]. The histopathology shows uninflamed cystic capsule surrounding a lumen lined by squamous or cuboidal epithelium of 1-4 cell layer thickness, which may exhibit some areas of thickening. These histopathological features show similarity with LPCs, and differential diagnosis between LPC and GCA should be supported by clinical and radiographic examinations to verify the extraosseous (GCA) or intraosseous (LPC) location. It is proposed that LPC arises from the proliferation of the dental lamina within the bone, while the GCA arises from the rests of the dental lamina in soft tissues [2]. Radiographic involvement in GCA is uncommon; however, it is reported in about $20 \%$ of the cases, and interestingly, it is sometimes confused with a LPC [4,5]. In these cases, strict clinicopathological correlation is necessary for establishing the correct diagnosis, because it is difficult to define on the panoramic radiograph alone whether the lesion is a GCA causing bone resorption

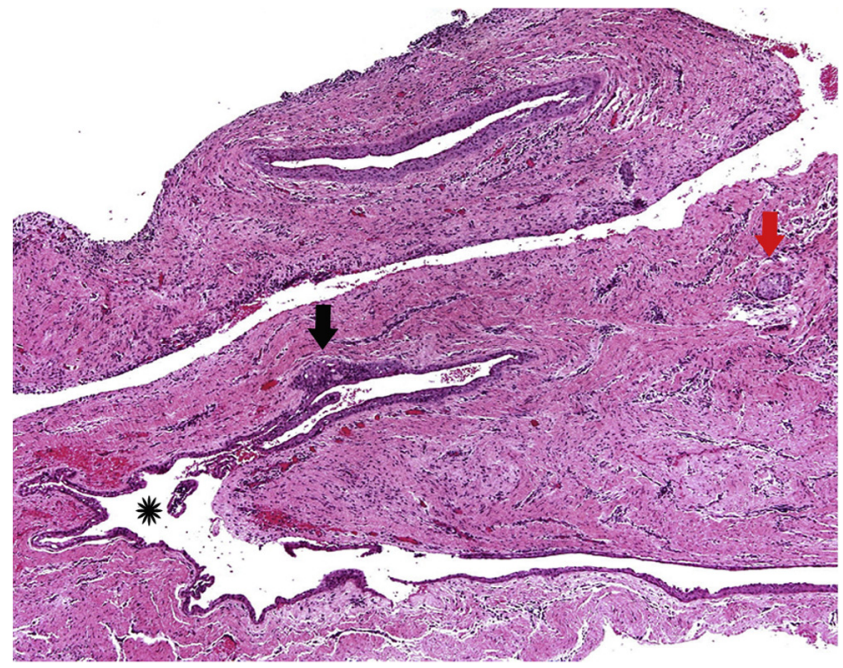

Fig. 3. Histopathological examination exhibiting a large cystic cavity lined by a thin stratified squamous nonkeratinized epithelium (black star) with foci of plaques or thickening of the epithelial lining (black arrow), as well as odontogenic epithelium (red arrow), were detected (H\&E; $\times 200)$. (For interpretation of the references to color in this figure legend, the reader is referred to the web version of this article.)

due to compression of the underlying bone or a LPC [4,5]. Interestingly, rare cases may occur in edentulous alveolar ridge of adult patients being the term ACA considered a synonym of GCA [6].

We report an unusual case presenting a nodular swelling on the edentulous maxilla with superficial bone resorption, which was diagnosed as a typical ACA.

\section{Funding}

No specific grant was received from funding agencies in the public, commercial, or not-for-profit sectors.

\section{Ethical approval}

The ethics committee approval was not necessary for this type of work.

\section{Conflicts of interest}

The authors declare no conflicts of interest.

\section{Acknowledgement}

Authors thank Dr. Alfredo Ribeiro-Silva for his contribution.

\section{References}

[1] Sato H, Kobayashi W, Sakaki H, Kimura H. Huge gingival cyst of the adult: a case report and review of the literature. Asian J Oral Maxillofac Surg 2007;19:176-8.

[2] Kelsey 5th WP, Kalmar JR, Tatakis DN. Gingival cyst of the adult: regenerative therapy of associated root exposure. A case report and literature review. J Periodontol 2009;80:2073-81.

[3] Wagner VP, Martins MD, Curra M, Martins MA, Munerato MC. Gingival cysts of adults: retrospective analysis from Two centers in South Brazil and a review of the literature. J Int Acad Periodontol 2015;17:14-9.

[4] Bell RC, Chauvin PJ, Tyler MT. Gingival cyst of the adult: a review and a report of eight cases. J Can Dent Assoc 1997;63:533-5.

[5] Tolson GE, Czuszak CA, Billman MA, Lewis DM. Report of a lateral periodontal cyst and gingival cyst occurring in the same patient. J Periodontol 1996;67:541-4.

[6] Speight P, Kessler H. Odontogenic and maxillofacial bone tumours. In: El-Naggar AK, Chan JKC, Grandis JR, Takata T, Slootweg P, editors. WHO classification of head and neck tumours. 4th ed.Lyon: IARC; 2017. p. 205-60. 